\title{
Broadband Performance Assessment of an RF Power Transistor Employing the Real Frequency Technique
}

This paper was downloaded from TechRxiv (https://www.techrxiv.org).

\section{LICENSE}

CC BY 4.0

SUBMISSION DATE / POSTED DATE

$10-01-2022$ / 12-01-2022

\section{CITATION}

yarman, siddik (2022): Broadband Performance Assessment of an RF Power Transistor Employing the Real Frequency Technique. TechRxiv. Preprint. https://doi.org/10.36227/techrxiv.18129218.v1

DOI

10.36227/techrxiv.18129218.v1 


\title{
Broadband Performance Assessment of an RF Power Transistor Employing the Real Frequency Technique
}

\author{
Sedat Kilinc, Malik E. Ejaz, B.S. Yarman, Life Fellow, IEEE, Serdar Ozoguz, Saket Srivastava and \\ Edmond Nurellari
}

\begin{abstract}
Generation of proper source/ load pull impedances for a selected active device is essential to design an RF power amplifier for optimum gain and power added efficiency. As they are obtained, these impedances may not be realizable network functions over the desired frequency band to yield the input and the output matching networks for the amplifier. Therefore, in this paper, first, we introduce a new method to test if a given impedance is realizable. Then, a novel "Real Frequency Line Segment Technique" based numerical procedure is introduced to assess the gain-bandwidth limitations of the given source and load impedances, which in turn results in the ultimate RF-power intake/ delivering performance of the amplifier. During the numerical performance assessments process, a robust tool called "Virtual Gain Optimization" is presented. Finally, a new definition called "Power-Performance-Product" is introduced to measure the quality of an active device. Examples are presented to test the realizability of the given source/load pull data and to assess the gain-bandwidth limitations of the given source/load pull impedances for a $45 \mathrm{~W}-\mathrm{GaN}$ power transistor, namely "Cree CG2H40045", over 0.8 -3.8 GHz bandwidth.
\end{abstract}

Index Terms - Immittance Realizability Conditions, Positive Real Functions, Foster Functions, Minimum Functions, Real Frequency Techniques, Broadband Matching, Gain-Bandwidth Limitation, Broadband Power Amplifier, GaN Transistor

\section{INTRODUCTION}

$\mathrm{F}^{\circ}$ OR wireless communication systems, it is essential to design power amplifiers (PA) for variety of applications [18]. Nowadays, it is a common practice to employ Gallium Nitrate $(G a N)$ transistors due to their high-power delivering capacity [9-13]. In practice, PA design process starts with careful selection of the power transistor considering the design parameters such as the required output signal power to be delivered, power added efficiency (PAE) of the amplifier, transducer power gain (TPG) over the specified bandwidth etc. Once the power transistor is selected, its nonlinear behavior is characterized by determining the optimum source-pull (SP) and

Sedat Kilinc is with Istanbul University-Cerrahpasa, and he is a Ph.D. student at Istanbul Technical University, Turkey. (e-mail: sedatkilinc@itu.edu.tr).

Malik Ejaz is a Ph.D. student at University of Lincoln, Brayford Pool, Lincoln LN67TS UK (e-mail: mejaz@lincoln.ac.uk).

B.S. Yarman is with Istanbul University-Cerrahpasa, Istanbul Technical University, Turkey and Lincoln University, Lincolnshire, UK, and RFT load-pull (LP) impedances. This process is achieved at each frequency by terminating the device with various impedance values, measuring the pre-set performance criteria such as gain and efficiency. The real and imaginary parts of the loading impedances are swept in a particular impedance zone on the Smith Chart to find the optimum source and load impedances. In practice, measured optimum source and load impedances are published in datasheets as in [14] One can easily make a narrow bandwidth PA design, per say, at a single frequency $f_{a}$ on the measured source and load pull impedance data. However, the design task becomes difficult if the bandwidth increases. For broadband PA design, we need to determine source and load pull impedance data at sufficiently large number of discrete frequencies to optimize the PA performance inside the frequency band of interest. In this case, the resulting source and load pull impedance data sets must correspond to realizable network functions, which eventually, yield optimum input and output matching networks, when they are modelled as positive real functions [15-18].

Let $Z_{S P a}\left(j \omega_{a}\right)=R_{S P a}\left(\omega_{a}\right)+j X_{S P a}\left(\omega_{a}\right)$ and $Z_{L P a}\left(j \omega_{a}\right)=$ $R_{L P a}\left(\omega_{a}\right)+j X_{L P a}\left(\omega_{a}\right)$ designate the discrete actual source and load pull impedance data at the actual angular frequency $\omega_{a}=$ $2 \pi f_{a}$ with actual frequency $f_{a}$. In these notations, subscript " $a$ " refers to measured actual values. To simplify the PA design process, measured impedance data is normalized with respect to a normalization resistance $R_{0}$ and a frequency $f_{0 a}$. In practice, $R_{0}$ may be selected as the standard termination $50 \Omega$ and $f_{0 a}$ can be chosen at the high end of the frequency band. In this case, normalized frequencies are designated by $f=\frac{f_{a}}{f_{a 0}}$, which is equal to normalized angular frequencies $\omega=\frac{2 \pi f_{a}}{2 \pi f_{0}}=$ $f$. Using the generic notation, a normalized impedance $Z(j \omega)$ is obtained by dividing the actual impedance $Z_{a}\left(j \omega_{a}\right)=$ $R_{a}\left(\omega_{a}\right)+j X_{a}\left(\omega_{a}\right)$ to $R_{0}$ such that $Z(j \omega)=\frac{Z_{a}}{R_{0}}=R(\omega)+$ $j X(\omega)$. Normalized frequencies are designated by $f$ or

Research Corporation of Teknopark Istanbul, Turkey. (e-mail: sbyarman@gmail.com).

Serdar Ozoguz is with Istanbul Technical University, Turkey, (e-mail: ozoguz@itu.edu.tr).

Saket Srivastava is with University of Lincoln, Brayford Pool, Lincoln LN67TS UK. (e-mail: ssrivastava@lincoln.ac.uk)

Edmond Nurellari is with University of Lincoln, Brayford Pool, Lincoln LN67TS UK. (e-mail: enurellari@lincoln.ac.uk) 


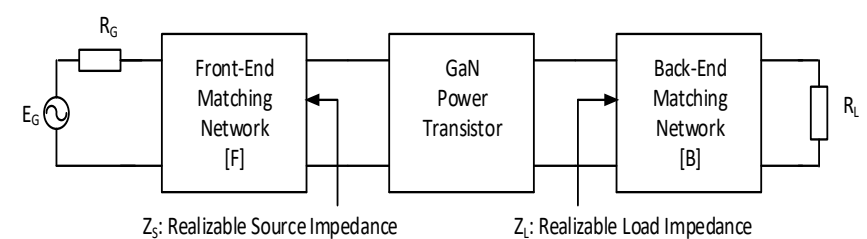

Fig. 1. A typical microwave power amplifier with realizable source and load impedances $Z_{S}$ and $Z_{L}$.

equivalently normalized angular frequencies is designated by $\omega=2 \pi f$. Likewise, normalized SP and LP impedances are represented by $Z_{S P}(j \omega)=R_{S P}(\omega)+j X_{S P}(\omega)$ and $Z_{L P}(j \omega)=$ $R_{L P}(\omega)+j X_{L P}(\omega)$ respectively. It is noted that the measured SP and LP terminations optimize the TPG as well as the PAE of the amplifier under consideration. Obviously, source and load pull impedances must be positive real (PR) functions so that one is able to analytically model the measured data and construct the front and the back-end matching networks as depicted in Fig. 1.

As they are placed on the Smith Chart, discrete source and load pull impedances may not describe positive real functions over broad frequency band. In this case, one must develop a test procedure to determine if $Z_{S P}$ and $Z_{L P}$ are PR functions. Eventually, the best possible-realizable source and load impedances must be generated to yield the front and the backend matching networks for the power amplifier to be designed. Therefore, in the following section, we introduce a novel numerical method to test the positive realness of a given discrete impedance data (Section II). Then, examples are given to test if a measured arbitrary immittance data and the source/load pull impedances obtained from the Cree's CG2H40045 GaN power transistor, represent positive real functions (Section III). In section IV, we propose a novel numerical process to assess the gain-bandwidth limitation of a given impedance data using the "Real Frequency-Line Segment Technique (RF-LST)" [19-21]. During the numerical performance assessments process, we introduce an original tool called "Virtual Gain Optimization (VGO)". In sections V and VI, "Gain-Bandwidth Limitations (GBWL)" of the source pull and the load pull impedances of the Cree CG2H-40045 GaN power transistor is determined over $0.8 \mathrm{GHz}-3.8 \mathrm{GHz}$ bandwidth respectively. In Section VII, we introduce a new definition to assess the Power-Intake and Power-Delivery performance of an active device. Finally, the paper is concluded in Section VIII.

\section{A Numerical ApProach To Test The Positive REALNESS Of THE MEASURED SP AND LP IMMITTANCE DATA}

While playing with immittance data on the Smith Chart, microwave engineers do not realize that the data placed on the Smith Chart with positive real part may not necessarily represent a realizable passive immittance function over the frequency band of interest. Therefore, one needs to test the data whether it is a positive real-realizable immittance or not.

Before we introduce the realizability test algorithm for the measured Source/Load Pull immittance data, let us review some of the related concepts of the network theory such as the definition of positive real (PR) functions, minimum functions, Foster functions, Hilbert transformation and Darlington's theorem. Detailed information on these topics can be found in major textbooks such as [21] and [22].

Let $p=\sigma+j \omega$ be the complex Laplace plane variable.

Definition 1: Any complex analytic function $K(p)$ is called positive real (PR) if and only if it is real for $p$ is real (i.e., for $p=\sigma, K(\sigma)$ is real) and $K(\sigma) \geq 0$ when $\sigma \geq 0$.

Definition 2: A positive real function $K_{m}(p)=\frac{a_{m}(p)}{b_{m}(p)}$, which is free of $j \omega$ poles, is called a "minimum function". In other words, $b_{m}(p)$ is strictly Hurwitz, which only posses Open Left Half Plane (O-LHP) zeros.

Definition 3: A positive real function $K_{F}(p)=a_{F}(p) /$ $b_{F}(p)$, which possess only "simple and interlacing $j \omega$ zeros and poles", is called a "Foster function".

Property 1: If $K(p)=\frac{a(p)}{b(p)}$ is $P R$, then it may be decomposed in to its minimum and Foster functions such that

$$
K(p)=K_{m}(p)+K_{F}(p)
$$

where

$$
K_{m}(p)=\frac{a_{m}(p)}{b_{m}(p)}=\sum_{i=}^{\tilde{n}} \frac{k_{i}}{p-p_{i}}
$$

with $p_{i}=\sigma_{i}+j \omega_{i}$ and $p_{i+1}=\sigma_{i}-j \omega_{i}$ with $\sigma_{i}<0$.

On the $j \omega$ axis, $K_{F}(p), K(p)$, and $K_{m}(p)$ are expressed as

$$
\begin{gathered}
K_{F}(j \omega)=j X_{F}(\omega) \\
K(j \omega)=R(\omega)+j X(\omega) \\
K_{m}(j \omega)=R(\omega)+j X_{m}(\omega)
\end{gathered}
$$

Property 2: For a Foster function $K_{F}(j \omega)=j X_{F}(\omega)$, derivative of $X_{F}(\omega)$ with respect to $\omega$ is always non-negative. In other words, The Foster reactance function $X_{F}(\omega)$ is a monotonically increasing function. That is, $\frac{d X(\omega)}{d \omega} \geq 0 ; \forall \omega$ with interlacing zeros and poles.

Theorem 1: A minimum function $K_{m}(j \omega)$ is uniquely determined from its real part $R(\omega)$ using Hilbert transformation such that

$$
X_{m}(\omega)=R_{\infty}+\frac{2 \omega}{\pi} \int_{0}^{\infty} \frac{R(y)}{y^{2}-\omega^{2}} d y=H\{R(\omega)\}
$$

For a minimum function $K_{m}(j \omega)$, the relation between its real part $R(\omega)$ and its imaginary part $X(\omega)$ is called the Hilbert transformation $(H T)$. In this paper, $H T$ operator is designated by $H\{$.$\} . Thus, we say that X_{m}(\omega)=H\{R(\omega)\}$.

\section{A. Numerical Evaluation of Hilbert Transformation}

The real part $R(\omega)$ of a minimum function may be piece-wise linearized as shown in Fig. 2. In Fig. 2, $R(\omega)$ is sampled at the break frequencies $\left\{\omega_{1}, \omega_{2}, \omega_{3}, \ldots \omega_{N}\right\}$ with corresponding break points $\left\{R_{1}, R_{2}, R_{3}, \ldots R_{N}\right\}$. Furthermore, it is assumed that adjacent sampled pairs $\left\{\omega_{j}, R_{j}\right\}$ and $\left\{\omega_{j+1}, R_{j+1}\right\}$ are connected by line segments. For a sufficiently large frequency placed at $\omega=\omega_{N}, R(\omega)$ becomes practically zero yielding $R_{N}=0$. Based on the line-segment representation of Fig. 2, $R(\omega)$ is simply evaluated using the following line equation. For $\omega_{j} \leq \omega \leq \omega_{j+1}$ such that $j=1,2, \ldots,(N-1), R(\omega)$ is given by 


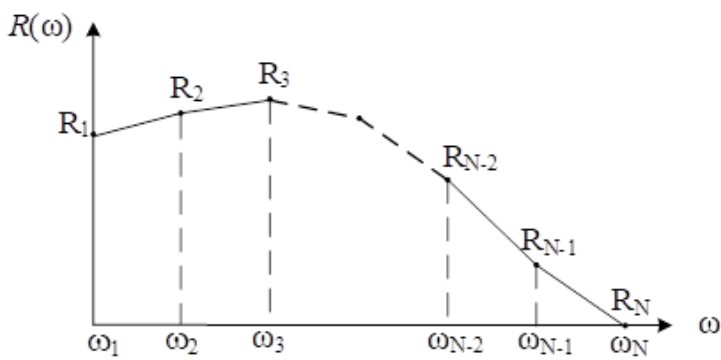

Fig. 2. Piecewise linearization of the Real part $R(\omega)$ of a minimum function $K_{m}(j \omega)=R(\omega)+j X_{m}(\omega)$.

$$
R_{j}(\omega)=a_{j} \omega+b_{j}
$$

where $a_{j}=\frac{R_{j}-R_{j+1}}{\omega_{j}-\omega_{j+1}}$ and $b_{j}=\frac{\left(R_{j+1}\right) \omega_{j}-\left(R_{j}\right) \omega_{j+1}}{\omega_{j-\omega_{j+1}}}$.

In the above representation of $R(\omega)$, we assume that $R_{\infty}=$ $\lim _{p \rightarrow \infty} R(\omega)$ is zero. This is a practical assumption for all passband amplifier designs.

Having line-segment representation of $R(\omega)$, imaginary part $X_{m}(\omega)$ of (3) is derived as

$$
X_{m}(\omega)=\sum_{j=1}^{N-1} \beta_{j}(\omega) \Delta R_{j} \triangleq H\{R(\omega)\}
$$

where $\Delta R_{j}$ and $\beta_{j}(\omega)$ is given by

$$
\begin{gathered}
\Delta R_{j}=R_{j+1}-R_{j} \\
F_{j}(\omega)=\left(\omega+\omega_{j}\right) \ln \left(\left|\omega+\omega_{j}\right|\right) \\
+\left(\omega-\omega_{j}\right) \ln \left(\left|\omega-\omega_{j}\right|\right) \\
\beta_{j}(\omega)=\frac{1}{\pi\left(\omega_{j}-\omega_{j+1}\right)}\left[F_{j+1}(\omega)-F_{j}(\omega)\right]
\end{gathered}
$$

It is noted that (4) and (5) are easily programmed in MatLab under the functions called "Line_Segment" and "Hilbert_Transform" respectively. Thus, one can generate a minimum function $K_{m}(j \omega)$ point by point from its real part $R(\omega)$ as it is specified in the form of line-segments without using any analytic forms. Resulting $K_{m}(j \omega)=R(\omega)+$ $j X_{m}(\omega)$ is for sure positive real. In the following sub-section, we propose a novel numerical procedure to decompose a positive real function into its minimum and Foster functions.

\section{B. Numerical decomposition of a measured immittance data to its minimum and Foster functions}

On the real frequency axis $j \omega$, (1a) can be regarded as a Theorem as follows.

Theorem 2: Any immittance data $K(j \omega)=R(\omega)+j X(\omega)$ can be decomposed into its minimum $K_{m}(j \omega)=R(\omega)+$ $j X_{m}(\omega)$ and Foster $K_{F}(j \omega)=j X_{F}(\omega)$ functions numerically such that

$$
K(j \omega)=R(\omega)+j X(\omega)=K_{m}(j \omega)+K_{F}(j \omega)
$$
where

$$
\begin{gathered}
X_{m}(\omega)=H(R) \\
X_{F}(\omega)=X(\omega)-X_{m}(\omega)
\end{gathered}
$$

with $\frac{d X_{F}(\omega)}{d \omega} \geq 0 ; \forall \omega$. Hence, (6) suggests the following algorithm to decompose "the measured immittance data" into its minimum and Foster functions. Then, one can test its positive realness by investigating the plot of the reactance curve
$X_{F}(\omega)$ if it is monotonically increasing.

It is crucial to note that the measured immittance data must be extrapolated over the entire frequency band to include $D C$ up to a frequency $f_{N}$ where the real part $R\left(\omega_{N}\right)$ is practically zero. Likewise, the imaginary part $X(\omega)$ is also extrapolated such that $X(0)=0$ and $X\left(\omega_{N}\right)$ is set to a reasonable value perhaps, by considering the immittance model, or in an ad-hoc manner to force $X_{F}(\omega)$ to become Foster reactance function if possible.

Based on the above derivations, let us introduce the following algorithm to separate the given immittance data to its minimum and Foster parts.

\section{Algorithm 1: Extraction of the Foster Reactance part from the measured immittance data \\ Inputs:}

$F A$ : An array which includes actual sampling frequencies $f_{i a}$ up to a point where the real part $R_{a}\left(f_{a}\right)$ practically vanishes and it is specified as a vector $F A=\left[f_{1 a} f_{2 a} \ldots f_{N a}\right]$. Note that it would be preferable to select $f_{1}$ at DC (i.e., $f_{1}=0$ ) by extrapolating the measured immittance data.

$R A$ : An array which includes actual sampled values of the real part of the measured immittance data $K_{a}\left(j f_{a}\right)=R_{a}\left(f_{a}\right)+$ $j X_{a}\left(f_{a}\right)$ at the actual frequencies $f_{a}$ and it is specified as a vector $R A=\left[\begin{array}{llll}R_{1 a} & R_{2 a} & \ldots R_{N a}\end{array}\right]$ (i.e. $R A$ includes all the break points) with $R_{N a}=0$.

$X A$ : An array that includes actual sampled values of the imaginary part of the measured immittance data $K_{a}\left(j f_{a}\right)=$ $R_{a}\left(f_{a}\right)+j X_{a}\left(f_{a}\right)$ at the actual frequencies $f_{a}$ and it is specified as a vector $X A=\left[\begin{array}{lll}X_{1 a} & X_{2 a} \ldots X_{N a}\end{array}\right]$. Most probably, at $D C$, $X_{1 a}=0$ for passive immittance data.

$f_{0}$ : Actual Normalization Frequency. It may be selected at the high end of the passband such that $f_{0}=f_{(N-1) a}$ where $R_{(N-1) a}>0$ with $R_{N}=0$ at $f_{N a}$.

$R_{0}$ : Actual Normalization Resistor. It may be selected as standard termination $50 \mathrm{ohm}$.

\section{Computational Steps}

Step 1: Augment and normalize the break frequencies $W B R=F A / f_{0}$ to cover $\omega=0$. The last frequency is located at a $\omega_{N}$ such that $R N=R\left(\omega_{N}\right) \cong 0$.

Step 2: Augment and normalize the real part $R(\omega)$ and $X(\omega)$.

Step 3: Compute $X m=$ Hilbert_Transform $(W B R, R)$ on the normalized real part $R(\omega)$.

Step 4: Compute the reactance function $X F(\omega)=X(\omega)-$ $X m(\omega)$.

Step 5: Plot $X F(\omega)$ against the normalized angular frequency $W$ and check if it is monotonically increasing. If $\left\{\frac{X F(\omega)}{d \omega} \geq 0 ; \forall \omega\right\}, X F(\omega)$ is Foster which in turn makes the measured immittance a positive real function.

Now, let us run an example to implement the above algorithm in the following section.

\section{ThE REAlizability TEST OF AN IMPEDANCE DATA}

The measured impedance data $Z A\left(j \omega_{a}\right)=R A\left(\omega_{a}\right)+$ $j X A\left(\omega_{a}\right)$ is depicted in Fig. 3. Measurements cover the 


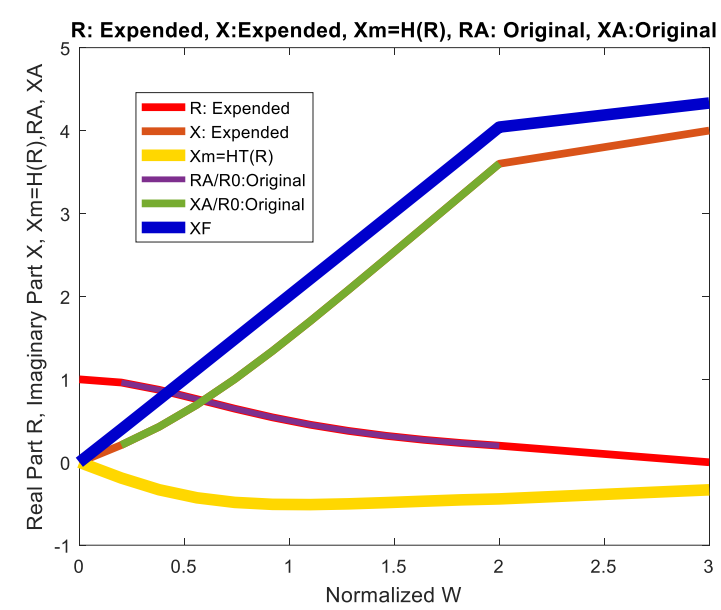

Fig. 3. Investigation of the measured impedance data if it is a positive real function. The answer is yes. It is PR.

frequency band of $f_{1 a}=0.2 \mathrm{GHz}$, and $f_{2 a}=2 \mathrm{GHz}$. Data is normalized with respect to $R_{0}=50 \Omega$ and $f_{0}=1 \mathrm{GHz}$. Test the measured impedance data whether it represents a positive real function.

Solution: For this purpose, we developed a MatLab program called "Main_Check_Immittance_Data_if_PR. $m$ ".

In this program, Algorithm 1 is implemented step by step using a total number of $N_{S}=11$ sampling points over 200 $\mathrm{MHz}-2 \mathrm{GHz}$ bandwidth. Now, let us implement the above Algorithm.

Step 1 Frequency Augmentation: Frequency sampling points is augmented by adding two more points at the low and the high ends. The first point is placed at $D C$, and the second point is selected at $f_{N a}=3 \mathrm{GHz}$ with $R_{N a}$ as shown in Fig. 3.

Step 2 Impedance Data Augmentation: The measured real part data $R A$ is gradually decreasing starting from $48 \mathrm{ohms}$ at $200 \mathrm{MHz}$. Then, it may be appropriate to set $R 1=R A(1)=$ $50 \Omega$ and the last break point is set to $R N=R A(N)=0$ at 3 $\mathrm{GHz}$. At DC, we assumed that the imaginary part $X(1)=0$. Notice that at $1.82 \mathrm{GHz}$, actual reactance is $X A(1.82 \mathrm{GHz})=160 \Omega$. At $2 \mathrm{GHz}, X A(2 \mathrm{GHz})=180 \Omega$. In other words, reactance $X$ is smoothly increasing towards $3 \mathrm{GHz}$. In this case, it may be reasonable to choose $X A$ as $X A(3 G H z)=200 \Omega$ or equivalently on the normalized scale, we set $X(3 G H z)=\frac{200}{50}=4$. Hence, augmented results are also shown in Fig. 3.

Step 3 Generation of the imaginary part $X_{m}(\omega)$ of the minimum function: This step is performed using our MatLab function $\quad X m=$ Hilbert_Transform $(W B R, R)$ which is programmed as in (5).

Step 4 Generation of Foster reactance function: This step is simply performed using the MatLab statement $X F=X-$ $X m$ as in (6).

Step 5: Plot the results and investigate the Foster reactance function $X_{F}(\omega)$ if it is monotonically increasing

Computed Foster reactance function $X_{F}(\omega)$ is also depicted in Fig.3. Close examination of this figure reveals that $X_{F}(\omega)$ is monotonically increasing. Therefore, the measured impedance data given by Fig. 3 belongs to a positive real impedance function. Therefore, it can be modelled as an analytic positive

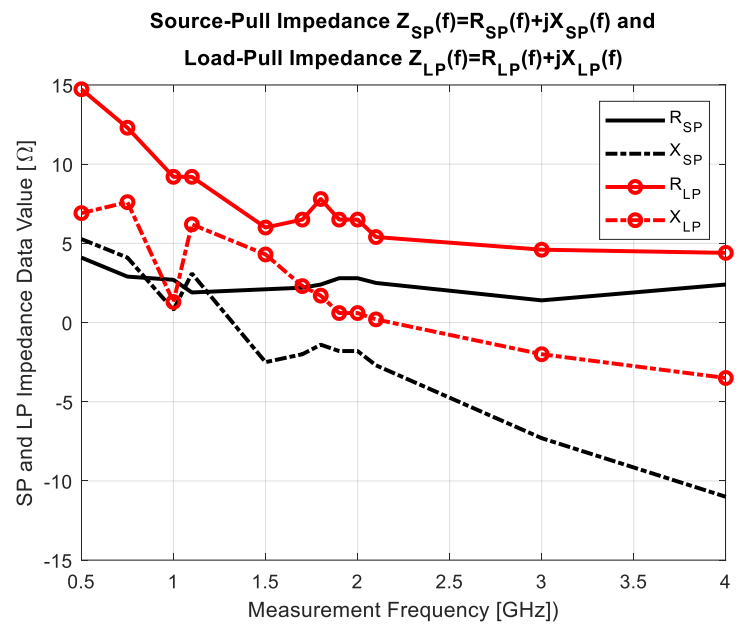

Fig. 4. Optimum Source-Pull and Load-Pull termination impedances.

real function without hesitation.

A. Examination of the Positive Realness of the Source and the Load Pull Impedances Obtained from a GaN Power Transistor

In this sub-section, we investigate the positive realness of the measured optimum source and load pull impedance data extracted from a GaN power transistor. The selected transistor is a Wolfspeed - CG2H40045, from Cree Company with nominal 45 Watts of output power [14]. As reported in [13], optimum source and load pull impedance plots are shown in Fig. 4. As described in the previous section, we extrapolated the given data to cover the frequencies $F 1 a=0(D C)$ and $F N a=$ $6 \mathrm{GHz}$. At DC, we select $R S P(1)=5 \Omega$ and $X S P(1)=0$. At $6 \mathrm{GHz}, \quad R S P(N)=0$ and $X S P(N)=-25 \Omega$ are selected. Similarly, load pull impedance data is augmented as $R L P(1)=$ $20 \Omega, R L(N)=0 \Omega$ and $X L P(1)=0, X L P(N)=-20 \Omega$.

Close examination of Fig. 5a reveals that, up to $500 \mathrm{MHz}$ (Band-I), the extracted Foster reactance $X_{F S P}(\omega)$ is monotonically increasing. Therefore, in this band, it behaves like a Foster function. Then, it decreases up to $1 \mathrm{GHz}$ (Band II). In this region it is non-Foster. From $1.0 \mathrm{GHz}$ to $2.0 \mathrm{GHz}$, it fluctuates such that over 1.0-1.1 GHz (Band III), 1.5-1.8 GHz (Band V) and 1.9-2.0 GHz (Band VII) it is a Foster reactance. However, over 1.1-1.5 GHz (Band IV), 1.8-1.9 GHz (Band VI) and after $2.0 \mathrm{GHz}$ (Band VIII), it shows non-Foster behavior. Referring to Fig 5b, computed Foster portion $X_{F L P}$ of the load pull impedance data is good from DC up to $750 \mathrm{MHz}$ (Band-1). It decreases between $0.75 \mathrm{GHz}$ and $1.0 \mathrm{GHz}$ (Band-2). In this region it is non-Foster. From $1.0 \mathrm{GHz}$ to $1.1 \mathrm{GHz}$ (Band-3) it increases. Therefore, in Band-3, it is Foster. After $1.1 \mathrm{GHz}$ (Band-4), $X F_{L P}(\omega)$ decreases monotonically (non-Foster). Thus, we say that measured load pull impedance data behaves like a proper positive real function in Bands 1 and 3 respectively. Otherwise it is non-Foster. So far, what we have observed that due to nonlinear behavior of the GaN transistorCree CG2H40045, reported source and load pull impedances are not realizable network functions over the frequency bands indicated above, even though they present positive real parts. However, they can be fitted to realizable network functions as much as possible, to construct the optimum front-end (input) 


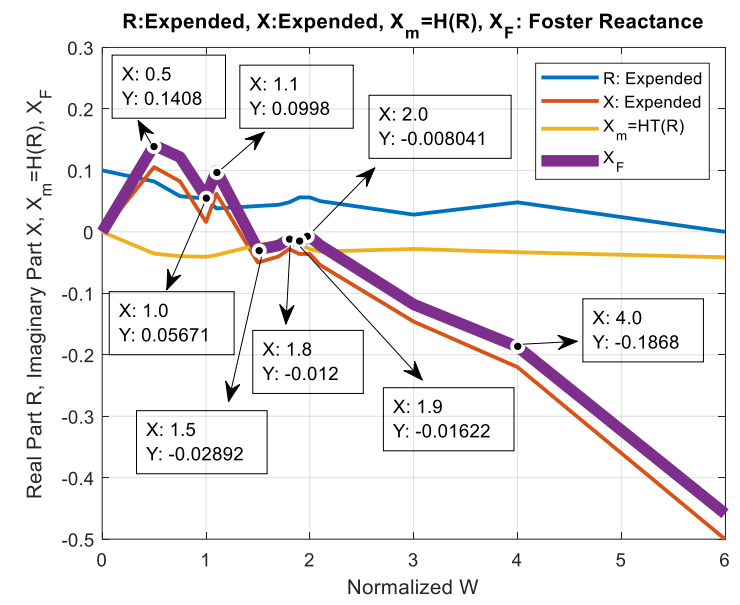

(a) Foster Part of the source pull impedance data.

Fig. 5. Extraction of the Foster Reactance from the source/load pull impedances.

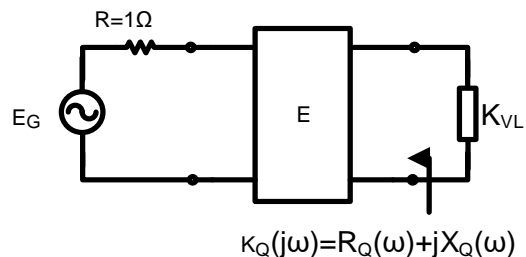

Fig. 6. A virtual single matching problem to model a measured immittance data $K(j \omega)$ by setting $K_{V L}=Z^{*}$.

and back-end (output) matching networks of the power amplifier. This process leads us to the gain-bandwidth limitation of the active device under consideration. In the follow up section, we propose an algorithm to assess the gainbandwidth limitation of the optimum source and the load pull impedances employing the real frequency line segment technique (RF-LST).

\section{NumericAl Assessment Of THE GAIN-BANDWIDTH LIMITATION OF A REALIZABLE IMMITTANCE}

The purpose of this section is to build the best realizable linesegment model for the reported source and load pulled immittances of [14] as much as possible. The proposed method is outlined as follows.

Let $K(j \omega)=R(\omega)+j X(\omega)$ refers to either the optimum source or load pull immittance to be modelled. In this regard, we define a virtual matching problem as shown in Fig. 6, where the virtual load $K_{V L}(j \omega)=R_{L V}(\omega)+j X_{V L}(\omega)$ is the complex conjugate of the immittance data to be modelled using the Real Frequency Line Segment technique [21]. In this case, the virtual $\operatorname{load} K_{V L}(j \omega)$ is expressed as

$$
\begin{gathered}
R_{L V}(\omega)=R(\omega) \\
X_{V L}=-X(\omega)
\end{gathered}
$$

Referring to Fig.6, in RF-LST, lossless matching network [E], is described by means of its PR driving point back-end immittance $K_{Q}(j \omega)=R_{Q}(\omega)+j X_{Q}(\omega)$ in Darlington sense. Furthermore, we assume that $K_{Q}(j \omega)$ is a minimum function. Therefore, $X_{Q}(\omega)$ is uniquely determined from $R_{Q}(\omega)$ via Hilbert Transformation as in (3). Referring to Fig. 2, the unknown of the matching problem is selected as the real part $R_{Q}(\omega)$ and it is expressed by means of its unknown break points

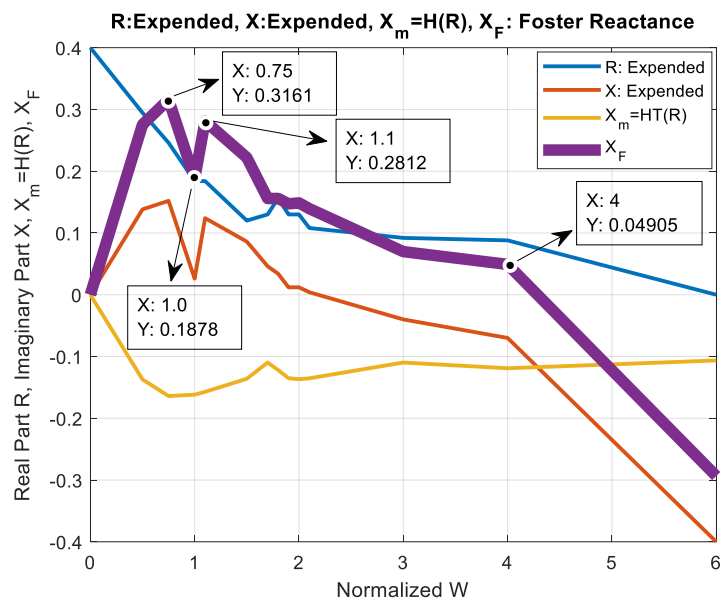

(b) Foster Part of the load pull impedance data.

$R Q A=\left[R_{1} R_{2} R_{3} \ldots R_{N-1} R_{N}\right]$, which is sampled at the break frequencies $W B R=\left[\omega_{1} \omega_{2} \omega_{3} \ldots \omega_{N-1} \omega_{N}\right] . \quad R_{Q}(\omega)$ is evaluated using (4) and $X_{Q}(\omega)$ is generated employing (5).

The unknown break points $\left[R_{1} R_{2} R_{3} \ldots R_{N-1} R_{N}\right]$ are determined to optimize the transducer power gain which is given by

$$
\begin{aligned}
T(\omega)=\frac{4 R_{Q} R_{L V}}{\left|K_{Q}(j \omega)+K_{L V}(j \omega)\right|^{2}} \\
=\frac{4 R_{Q} R_{L V}}{\left(R_{Q}+R_{L V}\right)^{2}+\left(X_{Q}+X_{L V}\right)^{2}} \\
=\frac{4 R_{Q} R}{\left[R_{Q}+R\right]^{2}+\left[H\left(R_{Q}\right)-X\right]^{2}}
\end{aligned}
$$

During the optimization process, we target a flat gain level $T_{0}$ to minimize the error function $\varepsilon(\omega)=T(\omega)-T_{0}$ over the frequency band of interest. The ideal solution is the unity TPG (i.e., $T(\omega)=1$ ) over passband, which yields $R_{Q}(\omega)=R(\omega)$ and $X_{Q}(\omega)=-X_{L V}=X(\omega)$ as desired. As $T(\omega)$ deviates from unity gain, the trace of line segment model shifts from the original data. Therefore, we say that "quality of immittance modelling" is measured by means of the transducer power gain of the virtual matching problem over the passband. During optimization, the error function may be expressed in terms of the unknown break points as

$$
\begin{aligned}
& \varepsilon(\omega)=T(\omega)-T_{0} \\
& =\frac{4 R_{Q} R}{\left[R_{Q}(\omega)+R(\omega)\right]^{2}+\left[\sum_{j=1}^{N-1} \beta_{j}(\omega) \Delta R_{j}-X(\omega)\right]^{2}}-T_{0}
\end{aligned}
$$

or equivalently,

$$
\begin{aligned}
& \varepsilon(\omega)=4 R_{Q} R-T_{0}\left\{\left[R_{Q}(\omega)+R(\omega)\right]^{2}\right. \\
&\left.\left.+\left[\sum_{j=1}^{N-1} \beta_{j}(\omega) \Delta R_{j}\right)-X(\omega)\right]^{2}\right\}
\end{aligned}
$$

In (9), $R(\omega)$ and $X(\omega)$ are the augmented immittance data to be modelled. $R_{Q}(\omega)$ is a line between the endpoints $\left(\omega_{j}, R_{j}\right)$ and $\left(\omega_{j+1}, R_{j+1}\right)$ for $\omega \in\left[\omega_{j}, \omega_{j+1}\right]$ as in (4).

It must be noted that $\varepsilon(\omega)$ is a quadratic/convex function in the 
unknown break points $R_{j}$. Therefore, the numerical minimization process is always convergent, and it is possible to hit global minimum of $\varepsilon$ yielding the "best solution for the break points with the highest value of the minimum of the passband gain" as shown in Fig. 7. Based on the last statement, the following sub-section is presented to assess the numerical gain bandwidth limitation of the given immittance data.

\section{A. The Best Transducer Power Gain T( $\omega)$}

Referring to Fig. 6 and Fig. 7, let $K(j \omega)=R(\omega)+j X(\omega)$ be the given immittance to be modelled as a realizable PR network function using the real frequency-line segment technique (RF-LST). Let $K_{Q}(j \omega)=R_{Q}(\omega)+j X_{Q}(\omega)$ be the RF-LST based minimum Driving Point Input Immittance (DPI) in Darlington sense. Let $T(\omega)$ be the transducer power gain (TPG) of the virtually matched system as specified by (8), which is optimized over the specified normalized angular frequency bandwidth $B$ such that $B=\left[\omega_{c 2}-\omega_{c 1}\right]$.

Let $T_{\min }$ be the minimum of $T(\omega)$ in $B$. Let $T_{\max }$ be the maximum of $T(\omega)$ in $B$. Let $T_{\text {mean }}=\frac{T_{\max }+T_{\min }}{2}$ be the mean value of $T(\omega)$ in $B$ and let $\Delta T=T_{\text {max }}-T_{\text {mean }}=T_{\text {mean }}-$ $T_{\min }$ be the gain fluctuation in $B$.

By trial and error, one can determine a flat gain level $T_{0}$ in such a way that $T_{\min }$ reaches to its maximum value in $B$. This state of TPG is called the "RF-LST based Gain BandwidthLimitation, or in short "RFLST-GBWL" of the given complex immittance $K(j \omega)$ over the specified bandwidth $B$. In this state, the mean value of the transducer power gain describes the average value of the power transfer with optimum fluctuations. In this state, $T P G$ may be expressed as $T(\omega)=$ $T_{\text {mean }} \mp \Delta T$.

During the minimization process of (9), $T_{0}$ may be swept starting from the flat gain level $T_{0}(1)=0.60$ upto to $T_{0}\left(n_{k}\right)=$ 1.00 with small step sizes $\delta T$. For example, $\delta T$ may be selected as $\delta T=0.05$. In this case, we define an index $k$ in a loop to minimize the error function $\varepsilon\left(\omega, T_{0}(k)\right)$ of (9) for a total number $n_{k}+1=9$ times. Then, at each step $k$, we store $K_{Q}(j \omega, k)=R_{Q}(\omega, k)+j X_{Q}(\omega, k), \quad T_{\min }(k), \quad T_{\max }(k)$, $T_{\text {mean }}(k), \Delta T(k) T(\omega, k)$ and $T_{0}(k)$ to determine the optimum "gain - state" yielding the "RFLST based GBWL" of the complex termination $K(j \omega)=R(\omega)+j X(\omega)$ over $B$, which in turn results in the best realizable-DPI in the form of line segments or equivalently as sampled data points.

For all the nonlinear optimization problems, initialization of the knowns is always crucial. Therefore, in the next subsection we present a rule of thumb to initialize the unknown break points $R_{j}$ for the minimization of the error function $\varepsilon\left(\omega, T_{0}(k)\right)$ for a fixed $T_{0}$.

\section{B. Initialization of the Nonlinear Minimization Process}

Before we introduce the optimization process, for a selected flat gain level $T_{0}$ and normalized break frequencies $\left\{\omega_{1}, \omega_{2}\right.$, $\left.\omega_{3}, \ldots \omega_{N}\right\}$, the unknown break points $\left\{R_{1}, R_{2}, R_{3}, \ldots R_{N}\right\}$ must be initialized. In this regard, initialization of the break points can be performed using (8) by assuming reactance cancellation. In other words, to maximize $T(\omega)$ of $(8)$, the reactance term

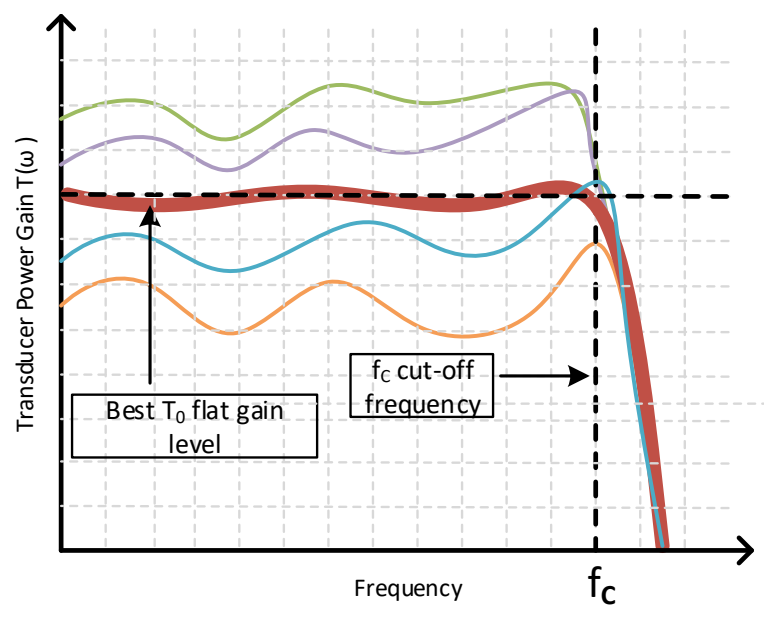

Fig. 7. Selection of the best GBWL by sweeping $T 0(k)$.

$\left(X_{Q}-X\right)$ is set to zero to derive the initials break points $R_{i n t-j}$ such that

$$
T_{0} \approx \frac{4 R_{Q} R}{\left[R_{Q}+R\right]^{2}}
$$

or

$$
R_{\text {int }-j}=R\left(\omega_{j}\right)\left[\frac{2-T_{0}+2 \mu \sqrt{1-T_{0}}}{T_{0}}\right] \geq 0
$$

In (10b), $\mu$ is a unimodular constant. It is set to $\mu=+1$ for the high values of initials $R_{i n t H-j}$ or it is selected as $\mu=-1$ for the low initials $R_{i n t L-j}$.

\section{Minimization of the error function: $\varepsilon\left(\omega, R_{1}, R_{2}, \ldots, R_{N-1}\right)$}

We use MatLab's nonlinear equation solver which is called "function lsqnonlin" to minimize the sum of square errors $\epsilon$ such that

$$
\epsilon=\sum_{r=1}^{N s}\left[\varepsilon\left(\omega_{r}, R_{1}, R_{2}, R_{3}, \ldots, R_{N-1}\right)\right]^{2} ; \omega_{c 1} \leq \omega_{r} \leq \omega_{c 2}
$$

In (11), the integer $N s$ is the total number of sampling points over the passband $B=\left[\omega_{c 2}-\omega_{c 1}\right]$ subject to optimization. The normalized angular frequencies $\omega_{c 1}$ is the low-end and $\omega_{c 2}$ is the high-end of the frequency band. Thus, the unknown break points $x=\left\{R_{1}, R_{2}, R_{3}, \ldots, R_{N-1}\right\}$ are determined to minimize the sum of squares error $\epsilon$. Minimization is performed using the Levenberg-Marquardt algorithm [23-25].

During minimization of sum of squares error, the first break point $R_{1}$ may be kept constant as it is initialized. This constant value may be zero for bandpass problems (i.e., $R_{1}=0$ ) or it may be included among the unknown break points. At the end of the minimization process, the realizable-driving point input immittance $K_{Q}(j \omega)=R_{Q}(\omega)+j X_{Q}(\omega)$ is obtained which is the optimum possible line-segment model for the measured immittance data $K(j \omega)=R(\omega)+j X(\omega)$.

Thus, we propose the following algorithm to assess the GBWL of the given immittance data. In the algorithm, the virtual matching problem of Fig. 6 is considered, and its virtual gain is optimized. This process is called the "Virtual Gain Optimization" or in short "VGO".

Algorithm 2: Determination of the GBWL of measured immittance data via VGO 


\section{Inputs:}

$F A$ : Actual frequency array which samples the immittance data to be modelled.

$R A$ : Measured real part of the immittance data. It is an array of the same size as $F A$.

$X A$ : Measured imaginary part of the immittance data. It is an array of the same size as $F A$.

$R 0$ : Normalization resistance.

F0: Normalization frequency.

$\omega_{C 1}$ : Low-end of the passband $\left(\omega_{C 1} \geq 0\right)$.

$\omega_{C 2}$ : High-end of the passband $\left(\omega_{C 2}>\omega_{C 1}\right)$.

$\omega_{s 1}$ : Low-end of the stop band.

$\omega_{s 2}$ : High-end of the stop band.

$N$ : Total number of break points $\left[R_{1}, R_{2}, R_{3}, \ldots, R_{N-1} R_{N}\right]$

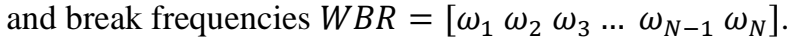

KFlag: This "integer" indicates if TPG is described in terms of impedances or admittances.

- $\quad$ if $K F l a g=1, T P G$ is impedance based.

- If $K F l a g=0, T P G$ is admittance based.

$R B 1$ : Initial value of the first break point. Note that one may select $R B 1=0$ for bandpass problems.

$n t r$ : Decide whether $R B 1 \geq 0$ is included among the unknowns or not. Choose $n t r=1$ if $R B 1$ is among the unknowns; otherwise set $n t r=0$.

$T_{01}$ and $T_{02}$ : Beginning and end levels of the flat gain $T_{0}(k)$ subject to optimization.

Note that in our programs we set $T_{01}=0.6$ and $T_{02}=1$. The reason for these choices is that, if $T P G$ is less than that of $T(\omega)<0.5$ (i.e. $T(\omega)$ is less than $-3 \mathrm{~dB}$ )., more than half of the available power is reflected back to the generator. In this case, termination immittance may be considered as "no good". In this case, the designer should seek a better power transistor.

$\delta T$ : Incremental step size to sweep the flat gain $T_{0}(k)$ from $T_{01}$ to $T_{02}$.

For our program, we select $\delta T=0.05$. Therefore, the index runs from 1 to $n k+1$ where $n k=\frac{\left(T_{02}-T_{01}\right)}{\delta T}=8$

$\mu$ (or sign): It is a unimodular constant. Decide whether you wish to work with high or low values of initial break points. Choose $\mu=+1$ for high value or set it to $\mu=-1$ for low value of initial break points.

\section{Computational Steps: Generation of the error function}

Step 1: Augment and normalize the given data points with respect to $R 0$ and $F 0$ as in Algorithm 1.

In this step, normalized complex immittance termination $K L A=(R A+j X A) / R 0$ is designated by its real part $R L A=$ $R A / R 0$ and its imaginary part $X L A=X A / R 0$ such that $K L A=$ $R L A+j X L A$ is a complex MatLab array. Its corresponding normalized break frequencies are designated by an array $W L A$ which includes augmented and normalized sampling frequencies such that $W L A=\left[\omega_{s 1}(F A / F 0) \omega_{s 2}\right]$ with, perhaps $\omega_{s 1}=0$ and $\omega_{s 2}=l \times \omega_{c 2}$ where $l$ is an integer and it may be selected as $1<l \leq 3$.

Step 2: Generation of break frequencies $W B R$ for the unknown break points.

- $\quad$ Set the first break frequency as $W B R(1)=W 1=$
$\omega_{S 1} \geq 0$

- $\quad$ Set the last break frequency as $W B R(N)=W N=\omega_{S 2}$ and distribute the remaining break frequencies between $\omega_{C 1}$ and $\omega_{C 2}$, perhaps uniformly. This step is performed using our MatLab function

$W B R=$ Break_Frequencies $(N, w s 1, w s 2, w c 1, w c 2)$

Step 3: For a selected flat gain level $T_{0}(k)$, generate the initials for the break points as in (10) such that

$$
\begin{gathered}
\omega_{j}=W B R(j) \\
R_{j}=R_{\text {int }-j}=R L A\left(\omega_{j}\right)\left[\frac{2-T_{0}(k)+2 \mu \sqrt{1-T_{0}(k)}}{T_{0}}\right] \geq 0 ; j \\
=1,2,3, \ldots, N-1
\end{gathered}
$$

Note that $R_{N}$ is fixed at zero (i.e., $R_{N}=0$ )

Step 4: Generate the imaginary part $X_{Q}(\omega)$ of the minimum function $K_{Q}(j \omega)$ as an array $X Q A$ using our MatLab function Hilbert_Transformation such that

$$
X Q A=\text { Hilbert_Transform }(W B R, R Q A)
$$

where $R Q A$ is an array which includes the all the unknown break points as they are initialized under $R Q A=$ $\left[R_{1} R_{2} R_{3 \ldots} R_{N}\right]$.

Step 5: Generate virtual $T P G$ as in (8) such that

$$
T(\omega)=\frac{4 R_{Q}(\omega) R(\omega)}{\left[R_{Q}(\omega)+R L(\omega)\right]^{2}+\left[X_{Q}(\omega)-X L(\omega)\right]^{2}} ; \quad \omega_{c 1}
$$

In this step, at any point $\omega=\omega_{i}$, values of $R_{Q}(\omega), R(\omega)$, $X_{Q}(\omega)$ and $X(\omega)$ are computed via linear interpolation, using our MatLab function $K_{i}=\operatorname{line} \_\operatorname{seg}\left(W, A, \omega_{i}\right)$ where the vector $A$ represents the generic break points of $R Q A, X Q A, R L A, X L A$ with the corresponding break frequency vector

and

$$
W=W B R \text { for }[R Q A \text { and } X Q A]
$$

$$
W=W L A \text { for }[R L A \text { and } X L A]
$$

Step 6: Generate the error function $\varepsilon\left(\omega_{i}\right.$, $\left.\left[R_{1} R_{2} R_{3 \ldots} R_{N}\right], T_{0}(k)\right)=T(\omega)-T_{0}(k)$ over the sampling points $\omega_{i}$ in the passband.

Step 7: Combine Step 2 through Step 6 with a nonlinear optimizer to determine the unknown break points $X=$ $\left[R_{1} R_{2} R_{3 \ldots} R_{N}\right]$.

It is noted that the above steps 2-6 are gathered under a MatLab function called

" Func

= error_CGH40F $(x, W B R, w c 1, w c 2, n t r, T 0, W L A, R L A, X L A) "$ where the vector $x$ includes all the unknown break points.

Step 8: Sweeping the flat gain level from $T_{01}=0.6$ to $T_{02}=$ 1.0 , repeat Step 2 through Step 7 for each flat gain levels $T_{0}(k)$ and at the end of the minimization process, store the following quantities.

- $\quad T_{-} \min (k)=$ Minimum of TPG in the passband.

- $\quad T_{-} \max (k)=$ Maximum of TPG in the passband.

- $\quad T_{-} \operatorname{mean}(k)=\left(T_{-} \max +T_{-} \min \right) / 2$

- $\operatorname{TPGA}(:, k)$ versus frequency

- $R Q A(:, k)$ versus frequency

- $X Q A(:, k)$ versus frequency 
Step 9: Find the best solution to determine the gainbandwidth limitation for the measured immittance data by generating the maximum value of the minimum gain $\operatorname{Tmin}=$ $\max \left(T_{\_} \min (k)\right)$ in the passband. Hence, RFLST based GBWL is determined using the MatLab commands of $[$ Tmin_Best,$r]=\max \left(T \_\min \right)$.

At this point, it is important to re-emphasize that the error function of (9b) is a convex function of the unknown break points $R_{i}$, regardless it's total numbers. Therefore, Algorithm 2 always hits the global minimum of the error function, which in turn yields the best solution for the selected flat gain level $T_{0}$ over the specified bandwidth. Thus, we say that RF-LST never requires to select a circuit topology nor an analytic form of a transfer function to optimize the transducer power gain of the matched structure under consideration. Hence, the ultimate gain-bandwidth limitation of the given source and load pull impedances are automatically determined. Eventually, optimum-realizable source/load pull impedances are obtained as the output of Algorithm 2.

The above algorithm is implemented under our MatLab program called

"Main_GBW_CG2H40045_SOURCEPULL_LST.m".

Now let us implement the above algorithm to generate the gain bandwidth limitations of the source and the load pull impedances for the Cree_CG2H40045 GaN power transistor.

\section{Gain BANDWidth Limitation OF THE SOURCE-PULL IMPEDANCE FOR CREE CG2H40045 GaN TRANSISTOR}

In this section, we will investigate the "power-intake" capability of the Wolfspeed/Cree_CGH240045 GaN transistor at its input [14]. Transistor is driven by a generator with $R_{0}=50 \mathrm{Ohm}$ internal resistance. The given source-pull impedance is approximated as the driving-point back-end impedance of a lossless two-port [E] when it is terminated in the complex-conjugate of the given source pull immittance as in Fig.5a. In this regard, it is desired to determine the gainbandwidth limitation of the given source-pull impedance as detailed in Algorithm 2. The given/augmented and the normalized source-pull impedances are depicted in Fig. 5a. User defined passband is specified over $f_{C 1}=800 \mathrm{MHz}$ and $f_{c 2}=3.8 \mathrm{GHz}$. In this case, we select the normalizing frequency $F_{0}$ at $F_{0}=3.8 \mathrm{GHz}$. The real part of the given source-pull impedance is augmented at DC (i.e., $f=0$ ) as $R S A(1)=5 \Omega$ and $R S A(N)=0 \Omega$. Similarly, the given imaginary part $X S A$ is augmented as $X S A(1)=0$ at $D C$ (i.e.ws $1=0$ ) and the end point is augmented at $6 \mathrm{GHz}$ as $X S A(N)=-25 \Omega$.

The GBWL of the virtual matching problem of Fig. 5a is numerically assessed via RF-LST employing our MatLab program "Main_GBW_CG2H40045_SOURCEPULL_LST.m". Inputs to the main program are summarized as follows.

$$
\omega_{C 1}=\frac{0.8}{F 0}=0.2105 ; \omega_{C 2}=\frac{3.8 \mathrm{GHz}}{F 0}=1 ; \omega_{s 1}=0.18 ; \omega_{s 2}=
$$

1.2. We have selected $N=21$ break points with 19 unknowns. In the optimization process, we prefer to work with impedances. Therefore, KFlag is set to 1 (i.e., KFlag =1). Furthermore, $n t r=0$ with fixed $R B 1=0$ located at DC, since we deal with

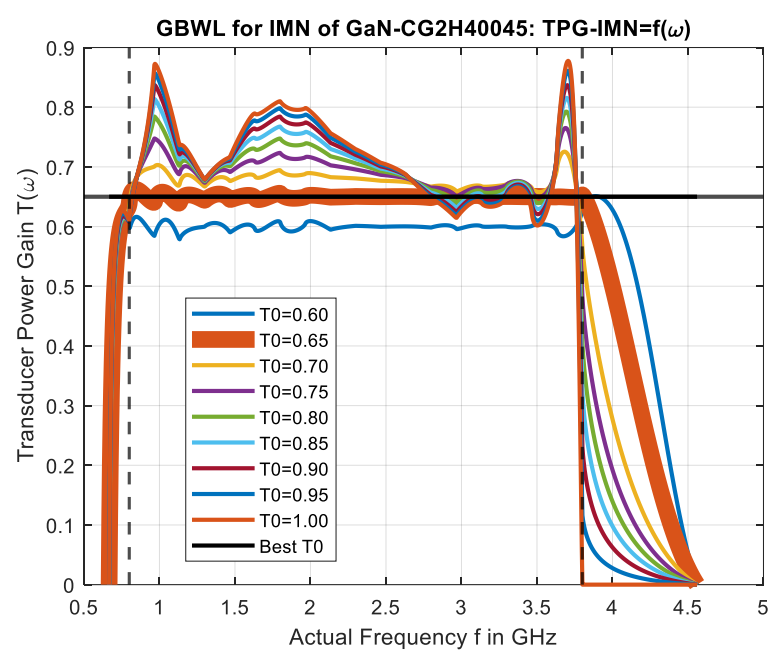

(a) GBWL by sweeping $T_{0}(k)$ obtained for the source pull data.

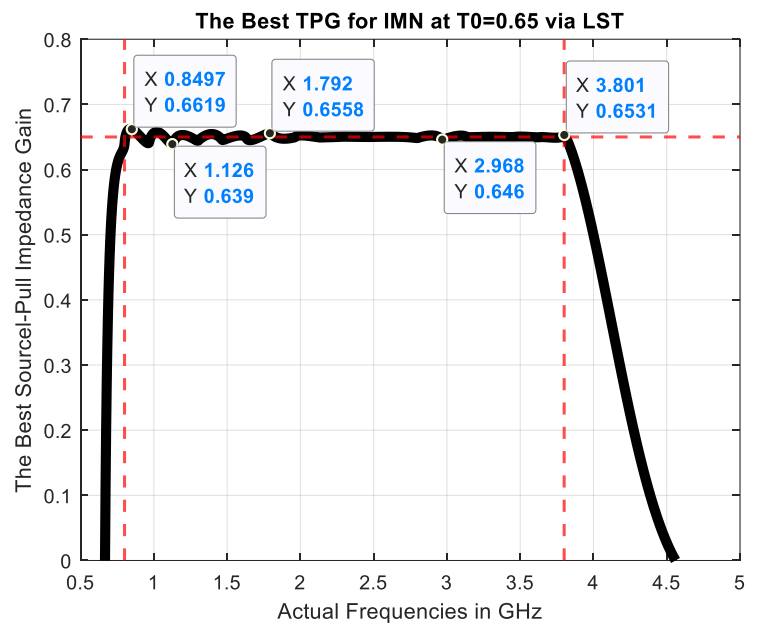

(b) The best TPG obtained for $T_{0}(7)=0.65$.

Fig. 8. GBWL for the optimum source pull data.

a bandpass single matching problem. Flat gain levels are swept between $\quad T 01=0.6$ and $\quad T 02=1.0$ with $\delta T=0.05$ incremental steps. Therefore, we define the total number of 9 flat gain levels starting from $T 0(1)=0.6$ up to $T 0(9)=1.0$. Eventually, we start the optimization with low initials. Therefore, $\mu=-1$ is selected.

Execution of "Main_GBW_CG2H40045_SOURCEPULL_LST.m" results in the RF-LST based Gain Bandwidth Limitation of the measured and augmented source pull impedance.

Results of the sequential optimization of TPG are summarized as in Table I and depicted as in Fig. 8. The RF-LST based Gain Bandwidth Limitation appears to be at $\max \left(T_{\min }\right)=0.6390$ with $r=2$, or equivalently, $T_{\text {mean }}=$ $0.6507 \mp 0.0117$. Optimized break points $R_{Q L S T-S P}$ and its corresponding Hilbert Transform $X_{Q L S T-S P}$ is depicted in Fig. $9 \mathrm{a}$ and $9 \mathrm{~b}$ respectively. Manufacturer published source pull impedances and RF-LST based source impedances are given in Fig. 10.

Referring to Table I and Fig. 8, it is predicted that $65.07 \%$ of the available power of the generator is transferable to the input of the power transistor $C G 2 H 40045$. In other words, GBWL of 


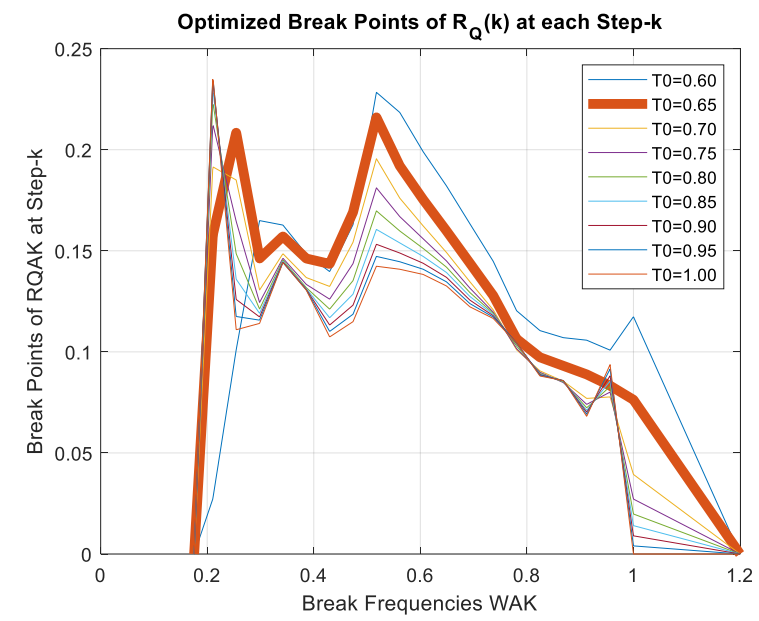

(a) Optimized $R_{Q A}(\omega)$.

Fig. 9. Optimized minimum $Z_{Q A}(j \omega)=R_{Q A}(\omega)+j X_{Q A}(\omega)$.

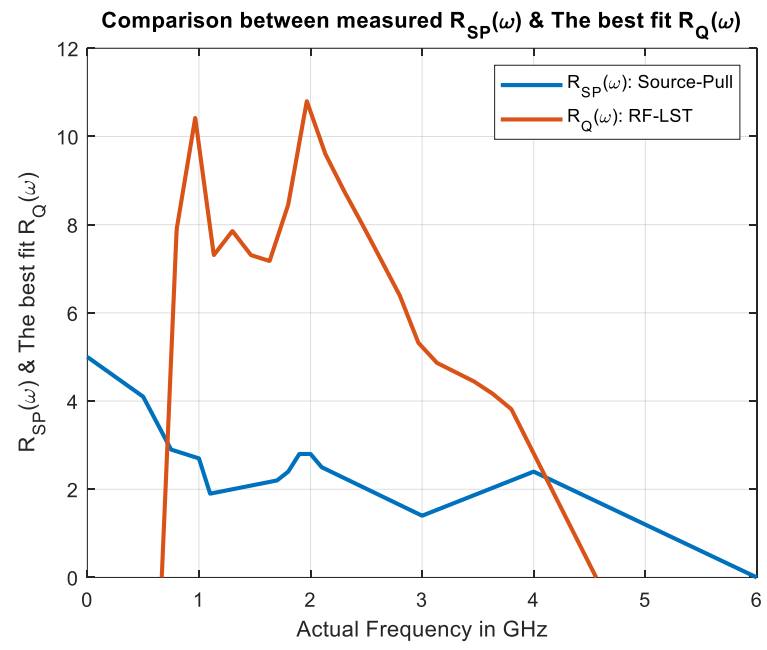

(a) RFLTS based RSP.

Fig. 10. Real and Imaginary parts of the original and the RFLST based source pull impedance.

TABLE I

GBWL OF SOURCE PUll IMPEDANCE OF CREe CG2H40045 POWER TRANSISTOR

\begin{tabular}{ccccccc}
\hline \hline$T_{0}(k)$ & $T_{\text {mean }}$ & $\begin{array}{c}F_{-} \max \\
(G H z)\end{array}$ & $T_{\max }$ & $\begin{array}{c}F_{-} \min \\
(G H z)\end{array}$ & $T_{\min }$ & $\Delta T$ \\
\hline 0.6000 & 0.5992 & 3.7930 & 0.6199 & 1.1339 & 0.5786 & 0.0206 \\
0.6500 & 0.6507 & 0.8419 & 0.6624 & 1.1261 & 0.6390 & 0.0117 \\
0.7000 & 0.6690 & 3.6840 & 0.7254 & 3.7852 & 0.6127 & 0.0564 \\
0.7500 & 0.6772 & 3.6879 & 0.7651 & 3.7813 & 0.5892 & 0.0880 \\
0.8000 & 0.6863 & 3.6957 & 0.7926 & 3.7774 & 0.5801 & 0.1062 \\
0.8500 & 0.6982 & 3.6996 & 0.8155 & 3.7735 & 0.5809 & 0.1173 \\
0.9000 & 0.7135 & 3.6996 & 0.8370 & 3.7697 & 0.5900 & 0.1235 \\
0.9500 & 0.7355 & 3.7035 & 0.8610 & 3.5049 & 0.6101 & 0.1254 \\
1.0000 & 0.7331 & 3.7074 & 0.8776 & 3.7658 & 0.5886 & 0.1445 \\
\hline
\end{tabular}

the optimum realizable source pull impedance is $T_{\text {mean }}=$ 0.6507 with optimum fluctuation of $\Delta T=\mp 0.0117$. Let us now investigate the output power delivering capacity of the same transistor using the load-pull measured impedance data in the following section. In this regard, it is desired to determine the gain-bandwidth limitation of the optimum load-pull impedance as detailed in Algorithm 2. The measured/augmented and the normalized load-pull impedances are depicted in Fig. 5b.

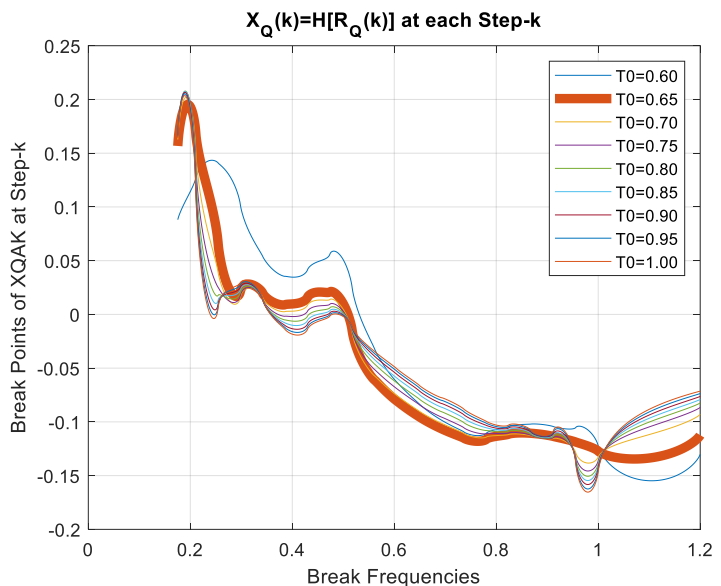

(b) $X_{Q A}(\omega)=H\left\{R_{Q A}(\omega)\right\}$.

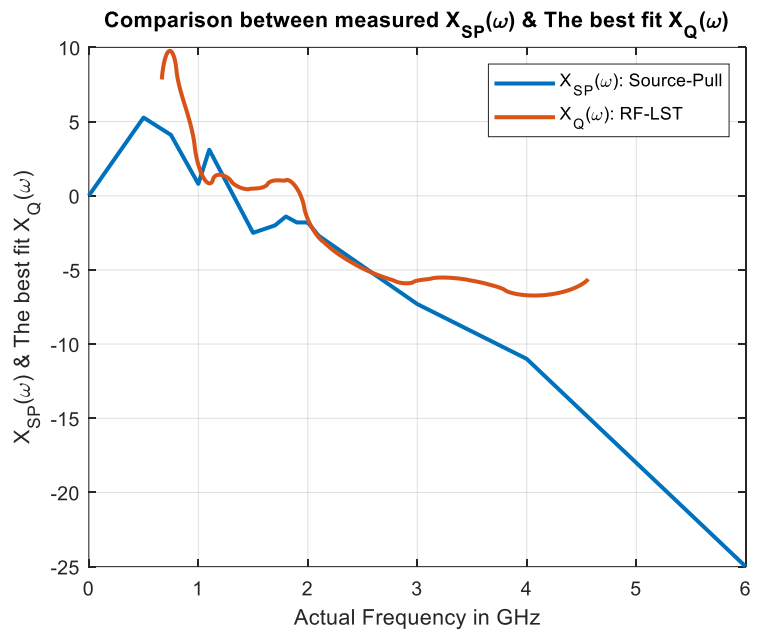

(b) RFLTS based XSP.

\section{GAIN BANDWIDTH LIMITATION OF THE LOAD-PULL IMPEDANCE FOR CREE CG2H40045 GaN TRANSISTOR}

In this section, we will investigate the power delivering capability of the Wolfspeed/Cree_CG2H40045 GaN transistor at its output-port [14]. Here, we assumed that the transistor is terminated in its modelled-realizable optimumload-pull impedance over the output matching network (OMN) while the other end of OMN is terminated in $R 0=50 \Omega$. The measured load-pull impedance is approximated as the drivingpoint backend impedance of a lossless two-port [E] when it is terminated in the complex-conjugate of the load pull immittance as in Fig. 5b.

As in the source-pull gain bandwidth limitation computations, user defined passband is specified over $f_{C 1}=$ $800 \mathrm{MHz}$ and $f_{c 2}=3.8 \mathrm{GHz}$. Therefore, we again set $F_{0}=$ $3.8 \mathrm{GHz}$. The real part of the measured load-pull impedance is augmented at DC (i.e., $f=0$ ) as $R L P A(1)=20 \Omega$ and $R L P A(N)=0 \Omega$. Similarly, the measured imaginary part $X L P A$ is augmented as $X L P A(1)=0$ at $D C$ (i.e.ws $1=0)$ and the end point is augmented at $6 \mathrm{GHz}$ as $X L P A(N)=-20 \Omega$.

The GBWL of the virtual matching problem of Fig. $5 \mathrm{~b}$ is 


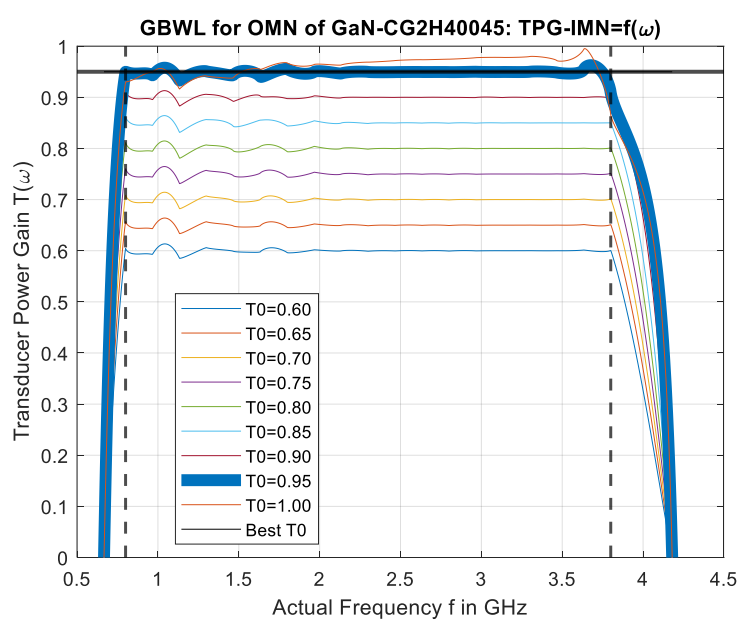

(a) GBWL of LP by sweeping $T 0(k)$.

Fig. 11. GBWL for the optimum load pull data.

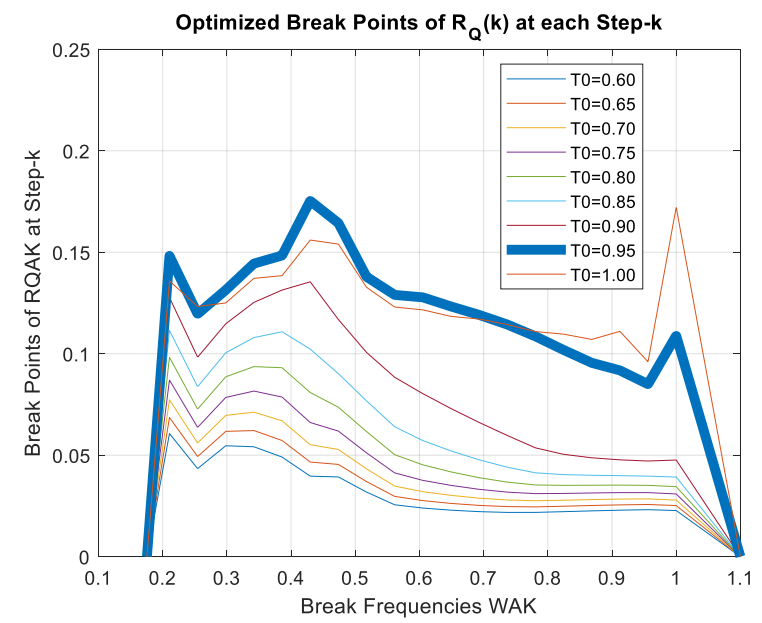

(a) Optimized $R_{Q A}(\omega)$.

Fig. 12. Optimized minimum $Z_{Q A}(j \omega)=R_{Q A}(\omega)+j X_{Q A}(\omega)$.

TABLE II

GBWL OF LOAD PULL IMPEDANCE OF CREE CG2H40045 POWER

TRANSISTOR

\begin{tabular}{ccccccc}
\hline \hline$T_{0}(k)$ & $T_{\text {mean }}$ & $\begin{array}{c}F_{-} \max \\
(G H z)\end{array}$ & $T_{\max }$ & $\begin{array}{c}F_{-} \min \\
(G H z)\end{array}$ & $T_{\min }$ & $\Delta T$ \\
\hline 0.6000 & 0.5992 & 1.0426 & 0.6133 & 1.1339 & 0.5851 & 0.0141 \\
0.6500 & 0.6487 & 1.0426 & 0.6639 & 1.1339 & 0.6335 & 0.0152 \\
0.7000 & 0.6982 & 1.0426 & 0.7143 & 1.1339 & 0.6821 & 0.0161 \\
0.7500 & 0.7479 & 1.0391 & 0.7646 & 1.1339 & 0.7312 & 0.0167 \\
0.8000 & 0.7977 & 1.0391 & 0.8146 & 1.1339 & 0.7809 & 0.0169 \\
0.8500 & 0.8478 & 1.0391 & 0.8642 & 1.1339 & 0.8313 & 0.0165 \\
0.9000 & 0.8980 & 1.0391 & 0.9130 & 1.1339 & 0.8829 & 0.0151 \\
0.9500 & 0.9478 & 3.6776 & 0.9628 & 1.1339 & 0.9328 & 0.0150 \\
1.0000 & 0.9557 & 3.6389 & 0.9952 & 1.1339 & 0.9162 & 0.0395 \\
\hline \hline
\end{tabular}

numerically assessed via RF-LST employing our MatLab program "Main_GBW_CG2H40045_LOADPULL_LST.m". Inputs to the main program is summarized as follows.

$\omega_{C 1}=\frac{0.8}{F 0}=0.2105 ; \omega_{C 2}=\frac{3.8 \mathrm{GHz}}{F 0}=1 ; \omega_{s 1}=0.18 ; \omega_{s 2}=$

1.1. We have selected $N=21$ break points with 19 unknowns. As in Example 2, in the optimization, we prefer to work with impedances (i.e., KFlag =1). Furthermore, $n t r=0$ with fixed $R B 1=0$ which is located at DC, since we deal with a bandpass single matching problem. The flat gain levels are swept between $T 01=0.6$ and $T 02=1.0$ with $\delta T=0.05$

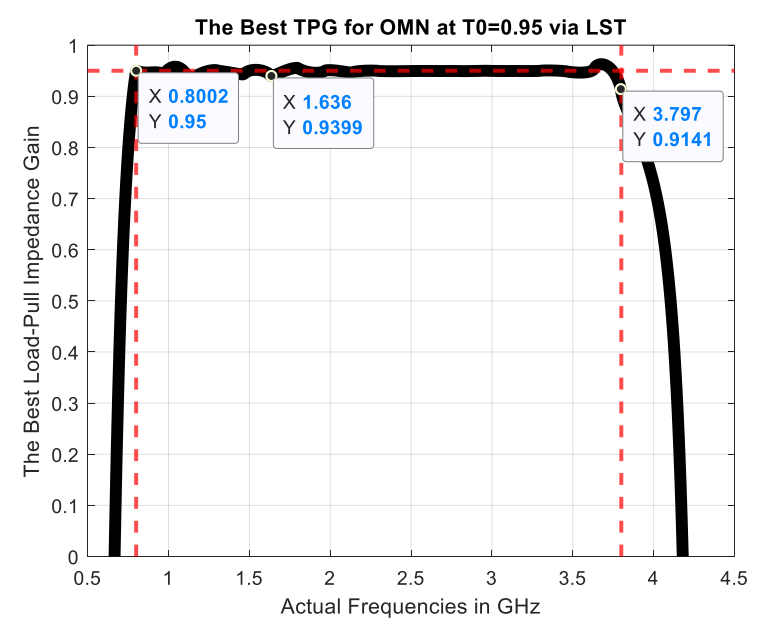

(b) The best TPG obtained for $T 0=0.95$.

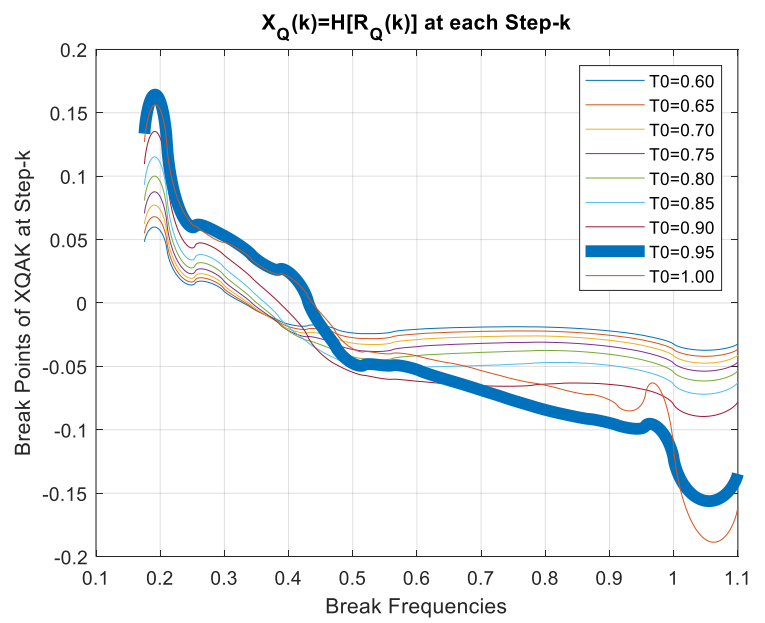

(b) $X_{Q A}(\omega)=H\left\{R_{Q A}(\omega)\right\}$.

increamental steps. Therefore, we define the total number of 9 flat gain levels starting from $T_{0}(1)=0.6$ up to $T_{0}(9)=1.0$. Eventually, we start the optimization with low initials. Therefore, $\mu=-1$ is selected. Execution of "Main_GBW_CG2H40045_LADPULL_RFLST.m" results in the RF-LST based Gain Bandwidth Limitation of the load pull impedance. The result of optimization is summarized as in Table II and depicted as in Fig. 11.

The RF-LST based Gain Bandwidth Limitation appears to be at $\max \left(T_{\min }\right)=0.9328$ or equivalently, $T_{\text {mean }}=$ $0.9478 \mp 0.015$ with corresponding loop index $r=8$.

The results of sequential optimization of $T P G$ is depicted in Fig. 12. Optimized break points $R_{Q L S T-L P}$ and its corresponding Hilbert Transform $X_{Q L S T-L P}$ is depicted in Fig. 12a and 12b respectively. Measured and modelled load pull impedances are given in Fig.13.

Referring to Table II and Fig. 11, it is predicted that $94.78 \%$ of the output power is delivered to the load pull impedance of the power transistor $C G 2 H 40045$. In other words, GBWL of the optimum realizable load pull impedance is $T_{\text {mean }}=0.9478$ with optimum fluctuation of $\Delta T=\mp 0.015$.

At this point it would be appropriate to define the power- 


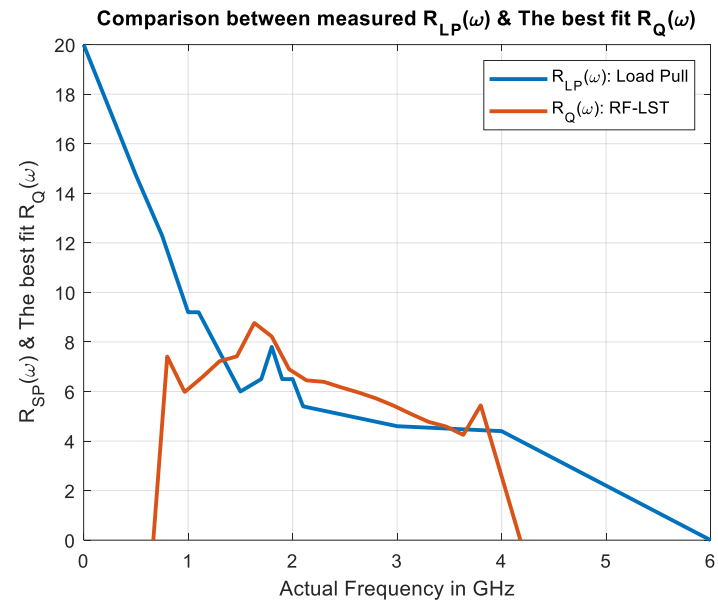

(a) RFLTS based RLP

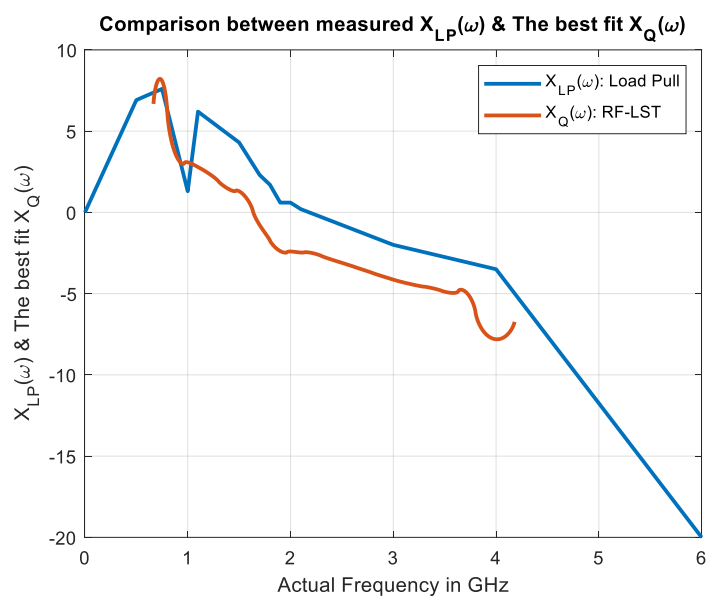

(b) RFLTS based XLP

Fig. 13. Real and Imaginary parts of the original and the RFLST based load pull impedance.

intake and power-delivering performance of an active device since its optimum-realizable RFT based source and load impedances refer to the ac-power absorption and power delivery capacity.

\section{Power Performance Product of An Active Device}

Considering the results of Section V and Section VI, we can define a new quantity called "Average Power Performance Product $(P P P)_{\text {mean }}$ " of a power transistor such that

$(P P P)_{\text {mean }}=[G B W L]_{S P} \times[G B W L]_{L P}$

where $[G B W L]_{S P}$ is the gain bandwidth limitation of the measured source-pull impedance and $[G B W L]_{L P}$ is the gain bandwidth limitation of the measured load-pull impedance respectively. Thus, we can say that maximum power performance product $(P P P)_{\max }$ of an active device cannot exceed

$$
(P P P)_{\max }=\left[T_{\max }\right]_{S P} \times\left[T_{\max }\right]_{L P}
$$

where $\left[T_{\max }\right]_{S P}$ and $\left[T_{\max }\right]_{L P}$ the values of the maximum pass band gains which corresponds to $[G B W L]_{S P}$ and $[G B W L]_{L P}$ respectively.

By Table I and Table II, for Cree CG2H40045, $(P P P)_{\text {mean }}=0.6507 \times 0.9478=0.6167$ (i.e., $\left.\sim 61.7 \%\right)$ and $(P P P)_{\max }=0.6624 \times 0.9628=0.6378=63.8 \%$.

\section{CONCLUSION}

An RF power amplifier design process starts with the characterization of the selected active device. The active device may be a GaN power transistor. In this regard, the source/load pull impedances of the active device are generated to optimize the power added efficiency (PAE) as well as the transducer power gain (TPG) of the amplifier over the frequency band of interest. Eventually, based on generated source/load pull impedances the input and the output matching networks of the power amplifier is designed. At this point, it is crucial to note that for many practical situations, source and load pull impedances placed on the Smith Chart, do not necessarily belong to realizable positive functions (PR) over the entire frequency band. In this case, one must check if these impedances are realizable. If not, it is well known that power intake and power delivery performance of the active device may be penalized heavily. In this regard, the designer must evaluate the power transistor properly to decide whether it is worth using it or not. Therefore, in this paper, firstly we introduced a novel numerical method to test the given source and load pull impedances if they can be used as realizable as driving point input impedances for the input and the output matching networks. Then, we introduce a new numerical method to assess the "Gain-Bandwidth Limitations (GBWL)" of the given source and load pull impedances employing the real frequency line segment technique. In this context, gain bandwidth limitations of the given source and load pull impedances yield the powerintake and the power delivery capacity of the selected active device respectively. The proposed numerical assessment method utilizes our newly developed robust "virtual gain optimization" tool called VGO. VGO minimizes a convex error function by targeting its global minimum, which in turn yields the optimum-realizable source and load terminations of the nonlinear active device under consideration. Thus, ultimate power intake and power delivery capacity of the nonlinear active device is determined. Finally, a new definition is introduced to measure the power-intake and power delivery quality of an active device, so called "Power-PerformanceProduct (in short PPP or 3P) based on the (GBWL) of the given source/load pull impedances.

Employing Cree's CG2H40045 GaN transistor, we exhibit the implementation of the proposed immittance realizability test procedure step by step and the design of an "optimum performance power amplifier algorithm" using the real frequency line segment technique over $0.8 \mathrm{GHz}-3.8 \mathrm{GHz}$ bandwidth which ideally receives average of $65.07 \%$ of the available power of the resistive input excitation and delivers the $94.78 \%$ of the transistor's output power to a resistive load.

\section{REFERENCES}

[1] Li, Meng; Pang, Jingzhou; Li, Yue; Zhu, Anding, "Ultra-Wideband Dual-Mode Doherty Power Amplifier Using Reciprocal Gate Bias for 5G," IEEE 
Transactions on Microwave Theory and Techniques, vol. 10, no. 67, pp. 4246-4259, 14 August 2019.

[2] Yuji Komatsuzaki; Rui Ma; Shuichi Sakata; Keigo Nakatani; Shintaro Shinjo, "A Dual-Mode Bias Circuit Enabled GaN Doherty Amplifier Operating in $0.85-2.05 \mathrm{GHz}$ and $2.4-4.2 \mathrm{GHz}$," in 2020 IEEE/MTT$S$ International Microwave Symposium, LosAngeles (LA), USA, 2020.

[3] G. Formicone, J. Burger, J. Custer, R. Keshishian and W. Veitschegger, "A Study for Achieving High Power and Efficiency based on High Bias Operation in Cand X-band GaN Power Amplifiers," in IEEE Topical Conference on Power Amplifiers for Wireless and Radio Applications (PAWR), San Diego, CA, USA, 2018.

[4] A. Grebennikov, Radio Frequency and Microwave Power Amplifiers. Volume 1: Principles, Device Modeling and Matching Networks, Institution of Engineering and Technology, 2019.

[5] G. R. Nikandish, R. B. Staszewski and A. Zhu, "Broadband Fully Integrated GaN Power Amplifier With Minimum-Inductance BPF Matching and TwoTransistor AM-PM Compensation," IEEE Transactions on Circuits and Systems I: Regular Papers, vol. 67, no. 12, pp. 4211-4223, 2020.

[6] G. R. Nikandish, R. B. Staszewski and A. Zhu, "A Fully Integrated GaN Dual-Channel Power Amplifier With Crosstalk Suppression for 5G Massive MIMO Transmitters," IEEE Transactions on Circuits and Systems II: Express Briefs, vol. 68, no. 1, pp. 246-250, 2021.

[7] B. Liu, C. C. Boon, M. Mao, P. Choi and T. Guo, "A 2.4-6 GHz Broadband GaN Power Amplifier for 802.11ax Application," IEEE Transactions on Circuits and Systems I: Regular Papers, vol. 68, no. 6, pp. 2404-2417, 2021.

[8] K. Krishnamurthy, T. Driver, R. Vetury, J. Martin, "100 W GaN HEMT Power Amplifier Module with> 60\% Efficiency over 100-1000 MHz Bandwidth," in IEEE IMS, Anaheim, California, USA, 2010.

[9] T. Kikkawa, K. Joshin and M. Kanamura, "GaN Device for Highly Efficient Power Amplifiers," Fujitsu Sci. Tech. J., vol. 48, no. 1, pp. 40-46, 2012.

[10] S. Mizuno, F. Yamada, H. Yamamoto, M. Nishihara, T. Yamamoto and S. Sano, "Development of GaN HEMT for Microwave Wireless Communications," SEI TECHNICAL REVIEW, no. 74, pp. 71-74, 2012.

[11] T. Maier, V. Carrubba, R. Quay, F. v. Raay and O. Ambacher, "Active harmonic source-/load-pull measurements of AlGaN/GaN HEMTs at X-band frequencies," in 83rd ARFTG Microwave Measurement Conference, Tampa, FL, USA, 2014.

[12] K. Inoue, S. Sano, Y. Tateno, F. Yamaki, K. Ebihara, N. Ui, A. Kawano and H. Deguchi, "Development of Gallium Nitride High Electron Mobility Transistor for
Cellular Base Stations," SEI TECHNICAL REVIEW, no. 71, pp. 88-93, 2010.

[13] R. S. Pengelly, S. M. Wood, J. W. Milligan, S. T. Sheppard and W. L. Pribble, "A Review of GaN on SiC High Electron-Mobility Power Transistors and MMICs," IEEE Transactions on Microwave Theory and Techniques, vol. 60, no. 6, pp. 1764-1783, 2012.

[14] Wolfspeed a Cree Company, "GaN HEMT Transistor series," https://www.wolfspeed.com/cg2h40045, Durham, North Carolina, USA 27703, 2021.

[15] S. C. Cripps, "A THEORY FOR THE PREDICTION OF GaAS FET LOAD-PULL POWER CONTOURS," in IEEE IMS 1983, Boston, MA, USA, 1983.

[16] V. Teppati, A. Ferrero, M. Sayed, "Ch-13: Load- and source-pull techniques", in Modern RF and Microwave Measurement Techniques, Cambridge, UK, The Cambridge RF and Microwave Engineering Series, Cambridge University Press, 2013, pp. 346383.

[17] T Maier, V Carrubba, R. Quay, F. van Raay, and 0. Ambacher, "Active Harmonic Source-/Load-Pull Measurements of AIGaN/GaN HEMTs at X-Band Frequencies," in 83rd ARFTG Microwave Measurement Conference, Tampa, Florida, USA, 2014.

[18] J. Cusack, S. Perlow and B. Perlman, "AUTOMATIC LOAD CONTOUR MAPPING FOR MICROWAVE POWER TRANSISTORS," in IEEE IMS 1974, Georgia, USA, 1974.

[19] H. Carlin, "New approach to gain bandwidth problems," IEEE Trans. on CAS, vol. 24, no. 4, pp. 170-175, 1977.

[20] B. S. Yarman, "Chapter 1: Circuit Theory for Power Transfer networks, Section 1.18 Positive Real Functions," in Design of Ultra Wideband Power Transfer Networks, West Sussex, UK, Jhon Wiley \& Sons Ltd., 2010, pp. 25-33.

[21] B. S. Yarman, "Chapter 11: Modern Approaches to Broadband Matching Problems; Real Frequency Solutions," in Design of Ultra Wideband Power Transfer Networks, West Sussex, UK, Jogn Wiley \& Sons Ltd, 2010, pp. 539-586.

[22] H. J. Carlin and P. P. Civalleri, in Wideband Circuit Design,, New York, CRC Press, 1998.

[23] K. Levenberg, ""A Method for the Solution of Certain Problems in Least-Squares"," p. pp. 164-168, 1944.

[24] D. Marquardt, " "An Algorithm for Least-squares Estimation of Nonlinear Parameters"," Journal Applied Mathematics, vol. 11, p. 431-441, 1963.

[25] T. F. Coleman and Y. Li, ""An Interior, Trust Region Approach for Nonlinear Minimization Subject to Bounds"," vol. 6, pp. 418-445, 1996. 


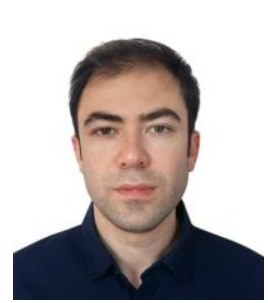

Sedat Kilinc received his BSc Degree from Yildiz Technical University, Istanbul (2012), MSc. Degree from Technical University of Istanbul (2015) in Electronics and Communications Engineering. He is currently studying towards his Ph.D. degree in Technical University of Istanbul. He has also been studying as a research assistant in Istanbul University Cerrahpasa since 2012. His research interests are power amplifier designs, semi-analytical techniques for wideband microwave power transfer network designs and synthesis with lumped/distributed circuit elements.

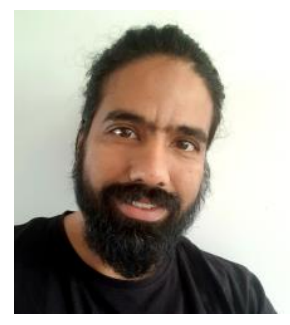

Malik Ehsan Ejaz received his BS in Computer Systems Engineering from SSUET Karachi Pakistan in 2004 and MSc in "Radio Systems Engineering" from The University of Hull in 2007. Currently, he is working towards his $\mathrm{PhD}$ in "A Novel Approach for Broadband and High-Efficiency Power Amplifier Design for 5G New Radio" from The University of Lincoln UK. Since the MSc he has worked as an $\mathrm{RF} \mid$ Microwave Engineer for various SMEs including X-Listed organizations in the UK and has been involved in the defense, medical, communication and RF heating related $R \& D$ and product development projects over the last fourteen years. His main contributions had been in the GaNSiC device development, GaNSiC device reliability test development, compound semiconductor device packaging, device characterization, communication jamming, UAV Tx/Rx module, power amplifiers, $\mathrm{RF}$ sources for medical devices, DDS based RF sources for jammers, plasma arching systems, RF power sensing and communication repeater design and development.

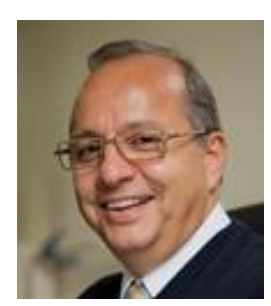

Binboga Siddik Yarman received his BSc Degree from Technical University of Istanbul (1974), MSc. Degree from Stevens Institute of Technology, Hoboken, N.J., USA (1977), and Ph.D. Degree from Cornell University (1982), Ithaca, NY, USA.

He was a Member of Technical Staff at General David Sarnoff Microwave Technology and Research Center (previously RCA Sarnoff Research Center), in Princeton, NJ, USA.

He served as professor and administrator at various Universities: Anadolu University, Middle East Technical University, Istanbul University, Isik University of Turkey; Ruhr University of Germany, Tokyo Institute of Technology of Japan, Wuhan Technology University of China and recently he is with Istanbul University, Technical University of Istanbul and the Lincoln University, UK.

$\mathrm{He}$ is one of the founders of Savronik Group of Companies and recently serves as the Chairman of the Board of Directors of Savronik International Co. He is the founding chairman of RFT Research \& Technology Corporation.
$\mathrm{He}$ is the inventor/co-inventor of many RF and Microwave Circuit Design Methods known as the "Real Frequency Techniques", speech, image and video encoding/decoding techniques known as "SYMPES" and the originator of the "Sympes-Phone" or in short "Symphone".

He was the founding president (1996-2004) and had served as the Chairman of Board of Trustees (2010- 2017) of Isik University.

He published more than 300 papers in the field of Microwave Engineering, Circuit and Systems, Signal Processing, Mathematical Modeling and Decision Making and six major research/text books. namely "Design of Ultra- Wideband Antenna Matching Networks" by Springer (2008), "Design of Ultra - Wideband Power Transfer Networks" by Wiley (2010), "Intelligence Based Decision Making" by Nobel Press of Turkey (2014), "Broadband Microwave and RF Power Amplifiers" by CRC-Taylor \& Francis (November 2016), "Design of Broadband RF and Microwave Power Amplifiers" by IET of UK (2020), "Design of Digital Phase Shifters for Multipurpose Communication Systems" by River Publisher (2020).

He holds 5 US and 9 Turkish Patents.

He was the recipient of Young Turkish Scientist Award of the Republic of Turkey (1986), Technology Award of the Scientific and Research Council of Turkey (1987). Research Award of Alexander von Humboldt Foundation of Germany (1987), He was selected as the "Man of the Year in Science \& Technology" by Cambridge Biography Center of UK (1998), Selected as the Fellow of IEEE (2004), member of the New York Academy of Science (1998).

$\mathrm{He}$ is the Life-Fellow of IEEE, Alexander Von Humboldt Fellow of Germany, and Salzburg Fellow of USIS (1985).

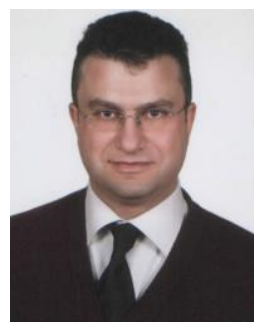

Serdar Ozoguz was born in Istanbul, Turkey. He received his B.Sc., M.Sc., and Ph.D. in Electronics Engineering from Istanbul Technical University in 1991, 1993 and 2000 respectively. Since 2009, he is working as a full professor in Istanbul Technical University. He is currently a full professor in electronics, teaching graduate and undergraduate courses. He is also the co-author of 150 papers published in scientific reviews or conference proceedings. His research interests include analog circuit design, chaotic circuits and chaos applications.

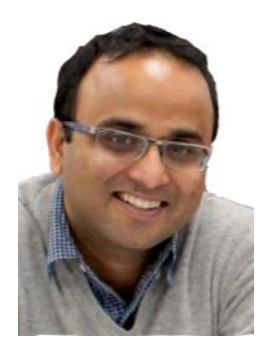

Saket Srivastava received the B.E. degree in Electrical and Electronics engineering from the National Institute of Technology, Tiruchirappalli, India, in 2003, and the Ph.D. degree in electrical engineering from the University of South Florida, Tampa, in 2008. He was a Postdoctoral Research Fellow in the School of Electronics and Computer Science, University of Southampton, U.K., from 2008 to 2010 and an Assistant Professor at the Indraprastha Institute of Information Technology, Delhi (IIITD), Delhi, India from 2010 to 2013. He currently a Senior Lecturer at University of Lincoln, UK. Dr. Srivastava serves on the technical program committees of 
several IEEE/ACM conferences. His research interests include embedded system design, FPGA design and synthesis, probabilistic modeling of emerging nanoelectronic devices, RF circuit design and software-defined radios.

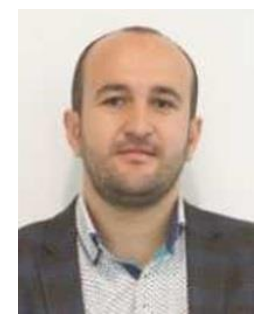

Edmond Nurellari Dr. Nurellari has been a faculty member with the School of Engineering at the University of Lincoln, United Kingdom, where he is currently an Associate Professor / Programme Leader in Electrical and Electronic Engineering / Robotics. His research interests include drone based communication systems, wireless power transfer, distributed signal processing, signal processing on graphs, resource allocations, distributed decisions and network security analysis in wireless sensor networks by employing tools from graph theory and game theory. 